\title{
RIEMANN SURFACES WITH AUTOMORPHISM GROUPS ADMITTING PARTITIONS ${ }^{1}$
}

ROBERT D. M. ACCOLA

A finite group $G_{0}$, is said to admit a partition if there is a set of subgroups $G_{i}, i=1,2, \cdots, s, s \geqq 2$, so that (1) $G_{0}=\bigcup_{i=1}^{s} G_{i}$ and (2) if $i \neq j$ and $i, j>0$, then $G_{i} \cap G_{j}=\langle e\rangle$ where $e$ is the identity in $G_{0}{ }^{2}$ Such groups are well known. ${ }^{3}$ The examples considered in this note are abelian groups in which every element has order two, and dihedral groups.

Let $W$ be a closed Riemann surface. A conformal self-map of $W$ will be called an automorphism. If $G$ is a finite group of automorphisms of $W$, then the orbit space, $W / G$, is naturally a Riemann surface and the natural projection $W \rightarrow W / G$ represents $W$ as an $n$-sheeted covering of $W / G$ where $n$ is the order of $G$.

The formula embodied in the following lemma is followed by some applications. The applications can be considered as generalizations and extensions of the hyperelliptic situation.

Lemma. Let $W$ be a closed Riemann surface of genus g. Suppose W admits a finite group of automorphisms, $G_{0}$, where $G_{0}$ is a group with a partition. Let the pertinent subgroups be $G_{1}, G_{2}, \cdots, G_{S}$. Let the order of $G_{i}$ be $n_{i}$, let $W_{i}=W / G_{i}$, and let $g_{i}$ be the genus of $W_{i}$ for $i=0,1$, $\cdots, S$. Then

$$
(S-1) g+n_{0} g_{0}=\sum_{i=1}^{S} n_{i} g_{i}
$$

Proof. If $r_{i}$ is the ramification of the $n_{i}$-sheeted covering $W \rightarrow W / G_{i}$ then the Riemann-Hurwitz formula for this covering is

$$
2 g-2=n_{i}\left(2 g_{i}-2\right)+r_{i} \text {. }
$$

Let $p(\in W)$ be a branch point in the covering $W \rightarrow W / G_{0}$. The elements of $G_{0}$ which leave $p$ fixed form a cyclic subgroup of $G_{0}$ generated by, say, $T .\langle T\rangle$ lies in the $G_{i}(i>0)$ containing $T$ (call it $G_{i_{0}}$ ) and no

Presented to the Society, November 22, 1967 under the title On cerlain groups of automorphisms of closed Riemann surfaces; received by the editors July 17, 1968.

${ }^{1}$ The research for this note was sponsored by the National Science Foundation (GP-7651).

${ }^{2}$ The notation $\langle S, T, \cdots\rangle$ will denote the group generated by $S, T, \cdots$.

${ }^{3}$ For a discussion of groups with partitions see Baer [1] and Suzuki [3]. 
subgroup of $\langle T\rangle$ lies in any other $G_{i}(i>0)$. Thus the contribution of $p$ to $r_{0}$ occurs in $r_{i_{0}}$ and in no other $r_{i}(i>0)$. It follows that

$$
r_{0}=\sum_{i=1}^{s} r_{i} .
$$

Moreover, a simple counting of elements shows that

$$
n_{0}=\sum_{i=1}^{S} n_{i}+1-S
$$

Formula (1) now follows by summing formula (2) as $i$ runs from 1 to $S$ and comparing the result to formula (2) with $i=0$ in the light of formulas (3) and (4). Q.E.D.

Formula (1) is of interest because the ramifications of the various coverings do not occur.

Application 1. Suppose $W$ is a Riemann surface of genus three which is a smooth two-sheeted covering of a surface $W_{0}$ of genus two. Then $W$ is hyperelliptic. ${ }^{4}$

Proof. $W$ admits an automorphism, $T$, of order two so that $W /\langle T\rangle=W_{0}$. Since $W_{0}$ is hyperelliptic, $W_{0}$ admits an automorphism, $S_{0}$, of period two so that the genus of $W_{0} /\left\langle S_{0}\right\rangle$ is zero. The composition of the two maps $W \rightarrow W_{0}$ and $W_{0} \rightarrow W_{0} /\left\langle S_{0}\right\rangle$ represents $W$ as a four-sheeted covering of the Riemann sphere. Since $W \rightarrow W_{0}$ is without ramification, the branch points of $W \rightarrow W_{0} /\left\langle S_{0}\right\rangle$ are all of order one and occur in pairs, one above the other. Since $W_{0} /\left\langle S_{0}\right\rangle$ is simplyconnected it follows that the covering $W \rightarrow W_{0} /\left\langle S_{0}\right\rangle$ arises from the action of a group of automorphisms, $G_{0}$, on $W$ where $G_{0}$ is isomorphic to the noncyclic group of order four. One element of $G_{0}$ is $T . G_{0}$ admits a partition into three subgroups of order two and so formula (1) applied to this situation gives

$$
g+2 g_{0}=g_{1}+g_{2}+g_{3} \text {. }
$$

Here $g_{0}=0$ and one $g_{i}$ must be two. Consequently another $g_{i}$ must be zero and so $W$ is hyperelliptic.

Application 2. If $W$ is a surface of genus three admitting an automorphism group, $G_{0}$, isomorphic to $Z_{2} \times Z_{2} \times Z_{2}$ then $W$ is hyperelliptic.

Proof. $G_{0}$ admits a partition with seven subgroups of order two. Formula (1) here yields

$$
3 g+4 g_{0}=\sum_{i=1}^{7} g_{i}
$$

- This fact was proved by H. M. Farkas [2] using theta functions. 
Since $g_{0} \geqq 0$ and $g=3$ one of the $g_{i}$ 's must be two and so $W$ is hyperelliptic by application one.

Application 3. If $W$ is a surface of genus five which is a smooth two-sheeted covering of a hyperelliptic surface of genus three, then $W$ is hyperelliptic or $W$ can be represented as a two-sheeted covering of a torus. The proof is exactly as in application one.

The proofs of the last applications require an examination of formula (1) applied to dihedral groups. Let $G_{0}$ be a dihedral group of order $2 n$. Let $R$ generate the cyclic subgroup of order $n$. If $V$ is an element of order two not in $R$, then $V_{t}=R^{t} V,(t=1,2, \cdots, n)$ are the elements of $G_{0}$ not in $\langle R\rangle$. If $G_{0}$ is realized as a group of automorphisms on a Riemann surface $W$, let $W_{R}=W /\langle R\rangle, W_{t}=W /\left\langle V_{t}\right\rangle, t$ $=1,2, \cdots, n, W_{0}=W / G_{0}$ with genera $g_{R}, g_{1}, \cdots, g_{n}$, $g_{0}$ respectively.

Formula (1) applied gives

$$
n g+2 n g_{0}=n g_{R}+2 \sum_{t=1}^{n} g_{t}
$$

If $n$ is odd, then all the groups $\left\langle V_{t}\right\rangle$ are conjugate. Thus the $W_{t}$ 's are all conformally equivalent and so $g_{t}=g_{1}$ for all $t=1,2, \cdots, n$. Thus we get

$$
g+2 g_{0}=g_{R}+2 g_{1} \text {. }
$$

If $n$ is even, then $\left\langle V_{t}\right\rangle$ is conjugate to $\left\langle V_{s}\right\rangle$ if and only if $t \equiv s(\bmod 2)$. Let $g_{1}$ and $g_{2}$ be the two genera in this case. Then,

$$
g+2 g_{0}=g_{R}+g_{1}+g_{2} \text {. }
$$

Actually formula (7) holds for $n$, odd or even, with $n$ odd implying that $g_{1}=g_{2}$. The similarity between formulas (7) and (5) accounts for the following applications. Of course, for $n=2$, formulas (5) and (7) are the same.

Application 4. Let $g$ be a nonnegative integer. Let $W^{\prime}$ be a Riemann surface of genus $g^{\prime}$ so that $g^{\prime}>4 g+1$. Suppose $S$ is an automorphism of $W^{\prime}$ of order two so that the genus of $W^{\prime} /\langle S\rangle$ is $g$. Then these properties define $S$ uniquely and $\langle S\rangle$ is central in the full group of automorphisms of $W^{\prime}$.

REMARK. This is a generalization of the hyperelliptic situation, $g=0$.

Proof. Suppose $S_{1}$ and $S_{2}$ are two distinct automorphisms of $W^{\prime}$ with the properties of $S$. Then, $S_{1}$ and $S_{2}$ generate a dihedral group, $G_{0}$, and we may assume $S_{1}=V_{1}$ and $S_{2}=V_{2}$. Formula (7) gives 


$$
g^{\prime}+2 g_{0}=2 g+g_{R} .
$$

The Riemann-Hurwitz formula applied to $W \rightarrow W /\langle R\rangle$ gives

$$
2 g^{\prime}-2=n\left(2 g_{R}-2\right)+r .
$$

But $n \geqq 2$ since $S_{1}$ and $S_{2}$ are distinct and $r \geqq 0$. So

$$
2 g^{\prime}-2 \geqq 2\left(2 g_{R}-2\right) \text { or } 2 g_{R} \leqq g^{\prime}+1 .
$$

Since $g_{0} \geqq 0$, we have

$$
2 g^{\prime}+4 g_{0}=4 g+2 g_{R} \leqq 4 g+g^{\prime}+1
$$

or

$$
g^{\prime} \leqq 4 g+1 .
$$

This contradiction shows that $S$ is unique.

Let $T$ be another automorphism of $W^{\prime}$. Then, $T^{-1} S T$ has the same properties as $S$. Thus $S=T^{-1} S T$, and the proof is complete.

Application 5. Let $g_{1}$ and $g_{2}$ be nonnegative integers. Let $W$ be a Riemann surface of genus $g$ so that

$$
2 g \geqq 3 g_{1}+3 g_{2}+3 .
$$

Let $W$ admit two distinct automorphisms $S_{1}$ and $S_{2}$, both of period two so that the genus of $W /\left\langle S_{i}\right\rangle$ is $g_{i}$. Then, $S_{1}$ and $S_{2}$ commute.

Proof. Let $G_{0}=\left\langle S_{1}, S_{2}\right\rangle$. Then $G_{0}$ is a dihedral group. Again set $S_{1}=V_{1}$ and $S_{2}=V_{2}$. Then,

$$
g+2 g_{0}=g_{1}+g_{2}+g_{R} .
$$

Let $R$ have order $n$. We wish to show that $n$ is two, so suppose $n \geqq 3$. The Riemann-Hurwitz formula for $W \rightarrow W /\langle R\rangle$ is

$$
2 g-2=n\left(2 g_{R}-2\right)+r
$$

or

$$
2 g-2 \geqq 3\left(2 g_{R}-2\right)
$$

or

$$
3 g_{R} \leqq g+2
$$

Since $g_{0} \geqq 0$ we have

$$
3 g \leqq 3 g+6 g_{0}=3 g_{1}+3 g_{2}+3 g_{R}
$$

or

$$
3 g \leqq 3 g_{1}+3 g_{2}+2+g .
$$


This contradicts the hypothesis and thus $n$ is two.

Application 6. Let $g$ be a positive integer. Let $W^{\prime}$ be a Riemann surface of genus $g^{\prime}$ so that

$$
g^{\prime}>3 g+2 .
$$

Suppose $W^{\prime}$ admits two distinct automorphisms $S_{1}$ and $S_{2}$, both with period two, so that the genus of $W^{\prime} /\left\langle S_{i}\right\rangle$ is $g, i=1,2$. Then $S_{1}$ and $S_{2}$ commute. Moreover, $\left\langle S_{1}, S_{2}\right\rangle$ and $\left\langle S_{1} S_{2}\right\rangle$ are normal in the full group of automorphisms of $W^{\prime} .^{5}$

Proof. That $S_{1}$ and $S_{2}$ commute follows immediately from the previous application. To show that $\left\langle S_{1}, S_{2}\right\rangle$ and $\left\langle S_{1} S_{2}\right\rangle$ are normal, we show that there is no further $S_{3}$ with the properties of $S_{1}$ and $S_{2}$. Thus conjugation will permute $S_{1}$ and $S_{2}$ and consequently leave $S_{1} S_{2}$ fixed.

Therefore, suppose that $S_{3}$ is a third distinct automorphism with the properties of $S_{1}$ and $S_{2}$. Consider $G_{0}=\left\langle S_{1}, S_{2}, S_{3}\right\rangle$. If $S_{3}=S_{1} S_{2}$ then $G_{0}$ is isomorphic to $Z_{2} \times Z_{2}$ and formula (5) leads to the contradiction

$$
g^{\prime}+2 g_{0}=3 g \text {. }
$$

Thus $G_{0}$ is isomorphic to $Z_{2} \times Z_{2} \times Z_{2}$. Let the seven subgroups of order two be denoted $G_{i}, i=1,2, \cdots, 7$, where $G_{i}=\left\langle S_{i}\right\rangle$ for $i=1,2,3$. Let $g_{i}$ be the genus of $W / G_{i}$. Formula (1) becomes

$$
3 g^{\prime}+4 g_{0}=\sum_{i=1}^{7} g_{i} .
$$

Now $g_{1}=g_{2}=g_{3}=g$. Also, as in Application 4, $2 g_{i} \leqq g^{\prime}+1$ for $i=4,5$, 6,7 . Multiplying equation (9) by two and putting in this information we get

$$
6 g^{\prime} \leqq 6 g^{\prime}+8 g_{0} \leqq 6 g+4 g^{\prime}+4 .
$$

This contradiction completes the proof.

REMARKs. We can conclude that if $g^{\prime}>3 g+2$ and $W^{\prime}$ admits an automorphism, $S_{1}$, of period two so that the genus of $W^{\prime} /\left\langle S_{1}\right\rangle$ is $g$, then $S_{1}$ is central or else there is one other automorphism, $S_{2}$, with the properties of $S_{1}$ and $S_{1} S_{2}$ is central. If $g^{\prime}>4 g+1$ Application 4 assures us that the first alternative holds. Simple examples show that these bounds are sharp. In fact, there is a surface, $W$, of genus five

- The author is indebted to W. 'T. Kiley for showing how the original form of Application 6 could be considerably strengthened to its present form. 
admitting a group of automorphisms isomorphic to $Z_{2} \times Z_{2} \times Z_{2} \times Z_{2}$ with five subgroups, $G_{i}$, of order two so that the genus of $W / G_{i}$ is one.

\section{REFERENCES}

1. Reinhold Baer, Partitionen endlicher Gruppen, Math. Z. 75 (1960/61), 333-372.

2. Hershel M. Farkas, Automorphisms of compact Riemann surfaces and the vanishing of theta constants, Bull. Amer. Math. Soc. 73 (1967), 231-232.

3. Michio Suzuki, On the finite group with a complete partition, J. Math. Soc. Japan 2 (1950), 165-185.

BROWN UNIVERSITY 